\title{
Pharmacological Manipulations of Anoxia-Induced Free Fatty Acid Accumulation in the Mouse Brain
}

\author{
Uffe Bang Olsen* and Aase Rand Kofod \\ Department of Pharmacology, CNS Division, Novo Nordisk A/S, Novo \\ Nordisk Park, DK-2760 Måløv, Denmark
}

Received April 3, 1991; Accepted July 24, 1991

\begin{abstract}
The accumulation of free fatty acid (FFA) in the brain occurs within minutes of anoxia, induced by exposing mice to a $100 \% \mathrm{~N}_{2}$ atmosphere. The rate of FFA release is high within the first minute and continues to increase moderately hereafter. FFA is apparently accumulated at the highest concentration in the cerebral hemispheres. The release of FFA can be inhibited partly by CNS depressants like N6-cyclopentyladenosine, pentobarbital, ethanol, or 4,5,6,7-tetrahydroisoxazolo-[5,4-c]pyridin-3(2H)-one (THIP). Antiadrenergic compounds such as reserpine, clondine, or prazosine were also found to be active. The $\mathrm{N}_{2}$ anoxia was initially and temporarily associated with motor excitation termed fight and flight reaction. This behavior could be reduced by administration of N6-cyclopentyladenosine, pentobarbital, ethanol, reserpine, and prazosine, but not by THIP or clonidine. The glutamate antagonist MK-801 inhibited the fight and flight reaction, but did not affect the FFA accumulation. The data are consistent with the view that brain anoxia initially increases FFA by receptor-mediated polyphosphoinositide breakdown and that the $\alpha-1$ adrenergic receptor is one of the receptors involved. The data also indicate that the fight and flight reaction is dissociated from the events that lead to FFA release, and may involve the stimulation of glutaminergic NMDA receptors.
\end{abstract}

*Author to whom all correspondence and reprint requests should be addressed. 
Index Entries: adenosine; cerebral ischemia; free fatty acids;

GABA; glutamate; motoric excitation; norepinephrine; PI hydrolyses.

\section{INTRODUCTION}

Originally, Bazán (1970) reported that brain ischemia and electroconvulsive shock increase brain free fatty acids (FFA). The enlargement of the pool is mainly owing to the production of arachidonic acid and stearic acid (Gardiner et al., 1981; Yasuda et al., 1985; Yoshida et al., 1980). The biochemical cascade is initiated by phospholipase C-mediated degradation of polyphosphoinositides (Abe et al., 1987; Huang and Sun, 1987; Ikeda et al., 1986; Yoshida et al., 1986) and diacylglycerol liberation is associated with elevated levels of FFA. A series of brain transmitters, including adrenergic $\alpha-1$ (Berridge, 1984), may utilize phosphatidylinositol hydrolysis in a second messenger system, and liberated FFA may eventually be involved in the development of postischemic neuronal damage via, e.g., lipid peroxidation or mitochondrial dysfunction (Hillered and Chan, 1988; Yoshida et al., 1980).

Biochemical changes related to experimental ischemia are frequently studied following decapitation or by surgical procedures (blood vessel occlusions). However, exposing animals to a $100 \% \mathrm{~N}_{2}$ atmosphere may be an alternative approach to inducing tissue anoxia and hence reproducing part of the ischemic situation. This procedure has been shown to decrease arterial $\mathrm{pO}_{2}$ to near zero within $1 \mathrm{~min}$ and to induce a temporary motor excitation termed fight and flight reaction (Wauquier et al., 1985). In the present study, FFA release in response to $\mathrm{N}_{2}$ anoxia has been quantified, and the effects of pharmacological test substances on FFA accumulation and motor excitation have been examined.

\section{METHODS}

Female NMRI mice $20 \pm 1 \mathrm{~g}$ were used for the experiments. The animals were not fasted and had free access to tap water.

Test compounds were usually dosed intraperitoneally (ip) and subsequently the animals were put in a cage with heated $\left(30^{\circ} \mathrm{C}\right)$ floor. The motor activity of the mice was inspected by a trained assistant before any anoxic challenge.

Some $30 \mathrm{~min}$ after drug administration the animals were placed individually in a small circular transparent plastic chamber into which a fast stream of $\mathrm{N}_{2}$ was introduced. After 10-15 sec of exposure to $100 \% \mathrm{~N}_{2}$ atmosphere the animals developed a vigorous motor activity termed fight and flight reaction (Wauquier et al., 1985) with the animals trying to escape the plastic chamber by jumping and circulating for $4-7 \mathrm{sec}$. This was 
followed by a state of unconsciousness in which the animals, while lying on their backs, continued to exhibit motor activity. The symptoms usually ended with a tonic seizure. The fight and flight reaction lasted 8-14 sec, and there was a clear positive correlation between the severity and the duration of the motor excitation. The response was scored as marked in untreated control mice, when animals showed the fight and flight reaction for more than $7 \mathrm{sec}$, moderate for less than $7 \mathrm{sec}$, and absent when the phenomenon was not observed. Respiratory movements terminated at ca. $45 \mathrm{sec}$. None of the control animals previously tested survived 1 min nitrogen exposure, at which time arterial $\mathrm{pO}_{2}$ was observed to be near zero (Wauquier et al., 1985).

At 1-min intervals after $\mathrm{N}_{2}$ exposure, the animals were removed from the chamber and were decapitated. The brains were immediately isolated and homogenized in $10 \mathrm{~mL} 96 \%$ ethanol in order to terminate enzymatic reactions. In order to minimize the ischemic period during brain excision, the tissue was not weighed. The mean wt of 10 separately isolated brains was $403 \pm 16 \mathrm{mg}$. From the time of decapitation to completed brain homogenization, 20-25 sec elapsed. To achieve final correction for FFA recovery (which usually ranged from 15-30\%), $\left[{ }^{14} \mathrm{C}\right]$ oleic acid $10,000 \mathrm{cpm}$ was added. The ethanol phase was isolated and evaporated. The FFA were further purified by phase partition at $\mathrm{pH}$ 3.0 and separated by monodimensional silica gel thin layer chromatography in the solvent mixture ethyl acetate:isooctane:acetic acid:water (55:25:10:50, the organic layer). The FFA at the front of the lanes was scraped off the plates and eluted with $2 \times 2.0 \mathrm{~mL}$ ethyl acetate. After evaporation the FFA was coupled to serum albumin by the addition of FFA-free rabbit serum (FFA had been removed by charcoal treatment). Subsequently FFA was determined spectrophotometrically by the acyl CoA synthetase- acyl CoA oxidase-peroxidase method (Mizuno et al., 1980) using a commercial kit (NEFAC, Code No.994-75409, Wako Chemicals, Neuss FRG). The calibration curve shows linearity over the range from $0.0-2.0 \mathrm{mmol} / \mathrm{L}$. Using our assay procedure, the lower limit of FFA detection is ca. $40 \mathrm{nmol} / \mathrm{brain}$.

On each test day, two to three animals per drug, and an equal number of controls, were examined. The results are presented as mean \pm S.E.M. The statistical significance of the results was assessed using Student's $t$-test unpaired comparison.

The following drugs were purchased: N6-cyclopentyladenosine (adenosine-A1 agonist) (cat.no C-8031) and yohimbine ( $\alpha-2$ antagonist) (cat.no Y-3125) from Sigma, St. Louis, MO, USA; pentobarbital sodium and reserpine from Mecobenzon, Copenhagen; THIP (4,5,6,7-tetrahydroisoxazolo-[5,4-c]pyridin-3(2H)-one) (GABA-A agonist) from Lundbeck a/s, Copenhagen; prazosine ( $\alpha-1$ antagonist) from Pfizer a/s, Copenhagen; clonidine ( $\alpha-2$ agonist) from Boehringer, Ingelheim FRG; and 


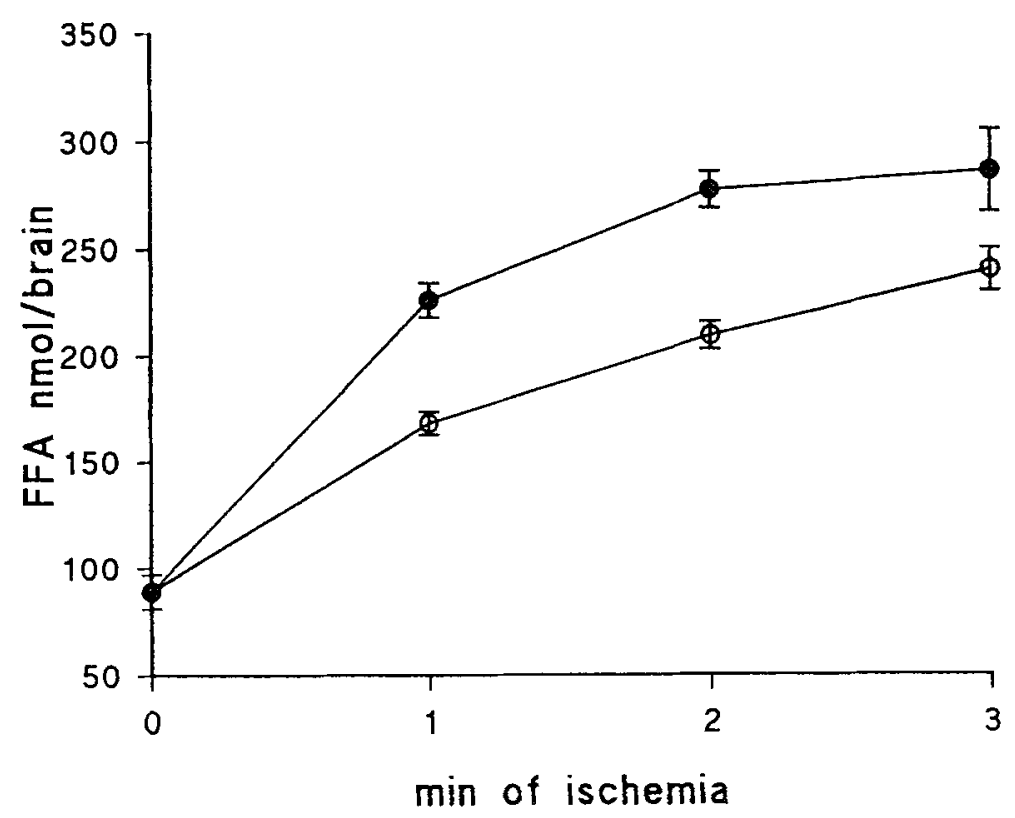

Fig. 1. Ischemia induced increase of FFA in relation to time. The results are mean $\pm \mathrm{S}$.E. of six to nine experiments. The $\mathrm{N}_{2}$ anoxia curve $(\bullet$ ) is significantly different from the decapitation ischemia curve $(0)$ at $\alpha=0.05$ (one way analyses of variance and multiple comparison of least square means).

MK-801 (NMDA antagonist) from MS\&D, Hertfordshire UK. Chemicals were of analytical grade from E. Merck, Darmstadt FRG.

\section{RESULTS}

Figure 1 shows the accumulation of FFA in the brain in relation to duration of anoxia. From this figure, it can be seen that FFA levels were significantly higher in animals that were killed by $\mathrm{N}_{2}$ anoxia than by decapitation. This discrepancy was evident within the first minute of anoxia, during which time the major changes in FFA concentration took place. Animals exposed to the $\mathrm{N}_{2}$ atmosphere exhibited motor excitation, fight and flight reaction (Wauquier et al., 1985).

In a small series of experiments the regional distribution of the accumulated FFA was determined after 2 min of $\mathrm{N}_{2}$-induced anoxia. In these experiments, the excised brains from 10 mice were quickly frozen on solid $\mathrm{CO}_{2}$. Subsequently, the brains were dissected into three major regions, the cerebrum, cerebellum, and the remainder (brain stem, etc.). The tissues were weighed frozen before extraction, and hence tissue FFA levels are presented as $\mathrm{nmol} / \mathrm{g}$ as follows: cerebrum $(658 \mathrm{nmol} / \mathrm{g})$, cerebellum $(541 \mathrm{nmol} / \mathrm{g})$, and the remainder $(459 \mathrm{nmol} / \mathrm{g})$.

Table 1 lists a series of general anesthetics and CNS depressive 


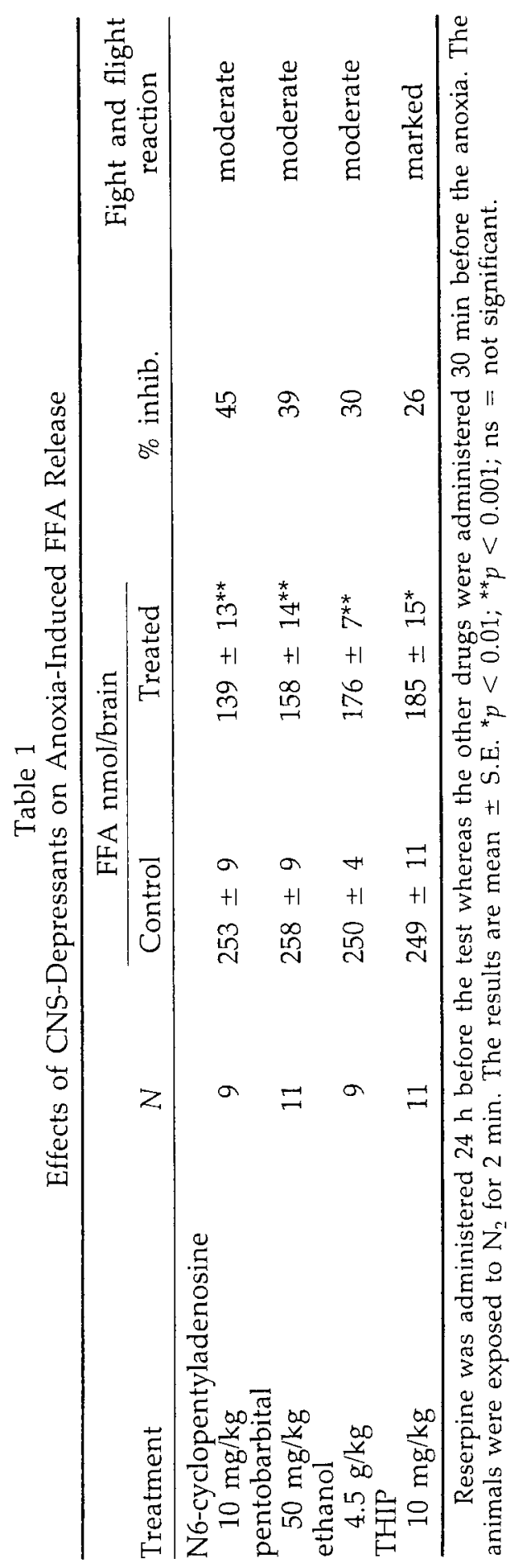




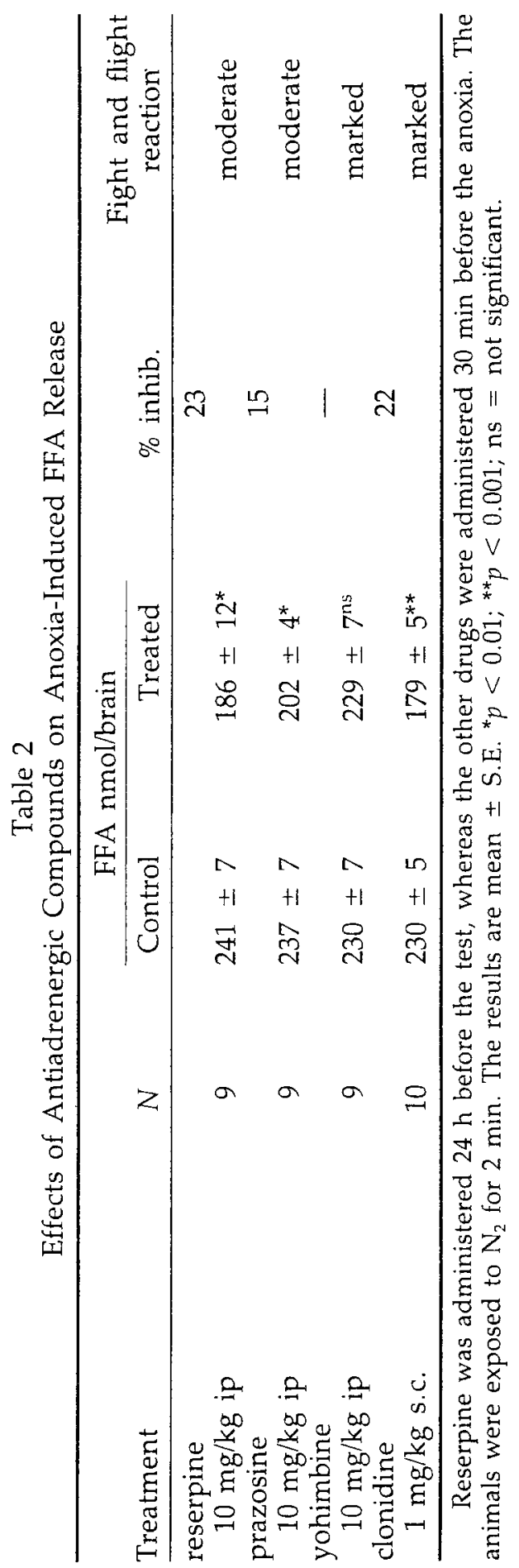


agents that were shown to significantly inhibit $2 \mathrm{~min}$ of $\mathrm{N}_{2}$ anoxiainduced brain FFA accumulation. It is noticeable that, in particular, N6cyclopentyl-adenosine exhibits a remarkable inhibitory efficacy. At the experimental doses used, these substances inhibited the motor activity before the anoxic challenge. With the exception of THIP, the drugs abolished the fight and flight reaction during the period of ischemia as judged by visual inspection.

Table 2 shows the inhibitory effects of several antiadrenergic compounds. It is demonstrated that the increase of FFA conc. was inhibited by reserpine and by the $\alpha-1$ antagonist prazosine, yet not by the $\alpha-2$ antagonist yohimbine. The table also indicates that clonidine inhibited anoxia-induced FFA release to a similar extent, as did reserpine pretreatment. With the exception of yohimbine, the compounds listed in Table 2 caused varying degrees of CNS depression, as indicated by decreased motor activity before the anoxic challenge. It is worth noticing that clonidine appeared not to decrease the fight and flight reaction during $\mathrm{N}_{2}$ exposure. In contrast, reserpine and prazosine showed such an activity.

MK-801 administered ip at a dose of $5 \mathrm{mg} / \mathrm{kg}(N=10)$ effectively inhibited the $\mathrm{N}_{2}$-induced fight and flight reaction, as this was absent in two of the animals and only moderate in the others. However MK-801 did not significantly inhibit the FFA accumulation during the first minute of anoxia (223 \pm 11 vs $197 \pm 14 \mathrm{nmol} /$ brain in controls and MK-801 treated animals respectively).

\section{DISCUSSION}

Like ATP, which decreases following anoxic challenge, an increase of FFA is one of the first biochemical markers to be changed in relation to ischemia (for review see Siesjö, 1984). Our data on brain FFA increase after decapitation are similar to those observed in previous studies (e.g., Bazán, 1970). The present "unstimulated" base-line level of mouse brain FFA (ca. $200 \mathrm{nmol} / \mathrm{g}$ ) is comparable to that $(323 \mathrm{nmol} / \mathrm{g}$ ) reported by Yasuda et al. (1985), in which the severed heads were immersed in liquid nitrogen, quickly frozen, and FFA was determined by gas chromatography. In addition, using the latter techniques, FFA levels of $96-146 \mathrm{nmol} / \mathrm{g}$ have been reported in rats (Yoshida et al., 1986; Ikeda et al., 1986). However, the present observation that $N_{2}$ anoxia initially raises FFA conc. levels at significantly faster rate than observed after decapitation is unique (Fig. 1). Moreover $\mathrm{N}_{2}$ anoxia is accompanied by a marked, yet transient, motor excitation that has been described as a fight and flight reaction (Wauquier et al., 1985).

We have observed that after $\mathrm{N}_{2}$-induced anoxia, FFA is accumulated throughout the brain, with relatively high concentrations in the hemispheres. This may differ from the unstimulated situation in vivo where 
the use of appropriate rapid freezing techniques has suggested much lower levels of FFA in rat cerebral cortex than in the hypothalamic/brain stem areas (Bazán et al., 1971).

Table 1 lists a series of CNS depressants with more or less welldefined mechanisms of action. They all reduced the FFA accumulation induced by $\mathrm{N}_{2}$ anoxia. Adenosine analogs have been shown to promote inhibition of CNS activity at every level of its organization. N6-cyclopentyladenosine is a potent agonist for the adenosine-A1 receptor and by negative feedback on presynaptic $\mathrm{A} 1$ receptors, this nucleoside inhibits further neurotransmitter release (Fredholm and Dunwiddie, 1988; Ribeiro, 1979), including that of norepinephrine (Fredholm, 1985).

THIP is a GABA-A agonist (Krogsgaard-Larsen et al., 1979) working at the GABA/chloride channel complex to depolarize the neuronal cell and hence decrease impulse traffic (Gold and Martin, 1984). The dose of THIP employed has been shown in other studies to exert near maximal anticonvulsive effect in mice subjected to audiogenic seizures (Meldrum, 1986). A general decrease of impulse traffic may also be achieved by the use of pentobarbital or ethanol in anaesthetic doses and may be expected to have an impact on the release of a series of brain transmitter substances, including those that subserve phosphatidylinositide hydrolyses as second messenger system. Our finding that pentobarbital inhibits the early rise of anoxia-induced FFA release accords with results from previous rat studies using decapitation ischemia (Hattori et al., 1986; Shiu et al., 1983).

Prazosine, an adrenergic $\alpha-1$ antagonist, produced a substantial inhibition of ischemia-induced FFA release (Table 2). This effect was not seen with the $\alpha-2$ antagonist yohimbine. This strongly indicates that central sympathetic outflow and subsequent $\alpha-1$ adrenergic stimulation may be important elements in the series of events that leads to FFA accumulation. This is further supported by the capacity of reserpine pretreatment to inhibit FFA increase. Apparently at variance with these results, Yoshida et al. (1984) reported comparable postdecapitation increases of FFA in intact and in locus ceruleus-lesioned (norepinephrine-depleted) rat hemispheres. However, the experimental designs are very different and moreover, major differences may normally exist between the two hemispheres in regard to ischemic FFA accumulation (Pediconi and Rodriguez de Turco, 1984). Prazosine and reserpine pretreatments also abolished the fight and flight reaction during the $\mathrm{N}_{2}$ anoxia (Table 2). This was in contrast to the adrenergic $\alpha-2$ agonist clonidine, which, however, was equally effective in inhibiting FFA release.

A dissociation between the fight and flight reaction and the $\mathrm{N}_{2}$ anoxia-induced FFA accumulation was also noted following administration of MK-801. This glutamate antagonist effectively inhibited the motor reaction to the $\mathrm{N}_{2}$-induced ischemia without significant effects on FFA release. MK-801 is a noncompetitive inhibitor of the NMDA receptor, 
which is coupled to the opening of calcium channels (Wong et al., 1986; Hüettner and Bean, 1988). Hence, the NMDA receptor may not be directly involved in phosphatidylinositide break-down and consequently an effect on early ischemic FFA release may not be anticipated. Indirectly however, our studies suggest that MK-801 might show some minimal inhibitory effect on FFA release. This could be owing to the stimulation of noradrenaline release in cerebral cortical slices by presynaptic NMDA receptors (Fink et al., 1990). Collectively, however, the results of the present study indicate that the fight and flight reaction in response to $\mathrm{N}_{2}$ anoxia may be dissociated from a simultaneous FFA release and may involve at least two transmitter systems, i.e., the adrenergic $\alpha-1$ and the glutaminergic NMDA.

\section{REFERENCES}

Abe K., Kogure K., Yamamoto H., Imazawa M., and Miyamoto K. (1987) Mechanism of arachidonic acid liberation during ischemia in gerbil cerebral cortex. J. Neurochem. 48, 503-509.

Bazán N. G. Jr. (1970) Effects of ischemia and electroconvulsive shock on free fatty acid pool in the brain. Biochim. Biophys. Acta 218, 1-10.

Bazán N. G. Jr., Pascual de Bazán H. E., Kennedy W. G., and Joel C. D. (1971) Regional distribution and rate of production of free fatty acids in rat brain. J. Neurochem. 18, 1387-1393.

Berridge M. J. (1984) Inositol trisphosphate and diacylglycerol as second messengers. Biochem. J. 220, 345-360.

Fink K., Bönisch H., and Göthert M. (1990) Presynaptic NMDA receptors stimulate noradrenaline release in the cerebral cortex. Eur. J. Pharmacol. 185, 115-117.

Fredholm B. B. (1985) Adenosine and central catecholamine neurotransmission, in Adenosine: Receptors and modulation of cell function (Stefanovich V., Rudolphi K., and Schubert J., eds.), pp. 91-104, IRL, Oxford.

Fredholm B. B. and Dunwiddie T. V. (1988) How does adenosine inhibit transmitter release? TIPS 9, 130-134.

Gardiner M., Nilsson B., Rehncrona S., and Siesjö B. K. (1981) Free fatty acids in the rat brain in moderate and severe hypoxia. J. Neurochem. 36, 1500-1505.

Gold M. R. and Martin A. R. (1984) $\gamma$-Aminobutyric acid and glycine activate $\mathrm{Cl}^{-}$channels have different characteristics in CNS neurones. Nature 308, 639-641.

Hattori T., Nishimura Y., Sakai N., Yamada H., Kameyama Y., and Nozawa Y. (1986) Attenuation by pentobarbital of free fatty acid and diacylglycerol liberation during global ischaemia in rat brain. Neurol. Res. 8, 33-38.

Hillered L. and Chan P. H. (1988) Role of arachidonic acid and other free fatty acids in mitochondrial dysfunction in brain ischemia. J. Neurosci. Res. 20, 451-456.

Huang S. F.-L and Sun G. Y. (1987) Acidic phospholipids, diacylglycerols, and free fatty acids in gerbil brain: a comparison of ischemic changes resulting from carotid ligation and decapitation. J. Neurosci. Res. 17, 162-167. 
Huettner J. E. and Bean B. P. (1988) Block of N-methyl-D-aspartate-activated current by the anticonvulsant MK-801: Selective binding to open channels. Proc. Natl. Acad. Sci. USA 85, 1307-1311.

Ikeda M., Yoshida S., Busto R., Santiso M., and Ginsberg M. D. (1986) Polyphosphoinositides as a probable source of brain free fatty acids accumulated at the onset of ischemia. J. Neurochem. 47, 123-132.

Krogsgaard-Larsen P., Hjeds H., Curtis D. R., Lodge D., and Johnston G. A. R. (1979) Dihydromuscimol, thiomuscimol and related heterocyclic compounds as GABA analogues. J. Neurochem. 32, 1717-1724.

Meldrum B. S. (1986) Preclinical test systems for evaluation of novel compounds, in New anticonvulsant drugs (Meldrum B. S. and Porter R. J., eds.), pp. 31-48, Libbey, London.

Mizuno K., Toyosato M., Yabumato S., Tanimizu I., and Hirakawa H. (1980) A new enzymatic method for colorimetric determination of free fatty acid. Anal. Biochem. 108, 6-10.

Pediconi M. F, and Rodriguez de Turco E. B. (1984) Free fatty acid content and release kinetics as manifestations of cerebral lateralization in mouse brain. $)$. Neurochem. 43, $1-7$.

Ribeiro J. A. (1979) Purinergic modulation of transmitter release. J. Theor. Biol. 80, 259-270.

Shiu G. K., Nemmer J. P., and Nemoto E. M. (1983) Reassessment of brain free fatty acid liberation during global ischemia and its attenuation by barbiturate anesthesia. J. Neurochem. 40, 880-884.

Siesjö B. K. (1984) Cerebral circulation and metabolism. J. Neurosurg. 60, 883-908.

Snyder D. S., Zimmerman T. R. Jr., Farooq M., Norton W. T., and Cammer W. (1983) Carbonic anhydrase, 5'-nucleotidase, and 2',3'-cyclic nucleotide-3'phosphodiesterase activities in oligodendrocytes, astrocytes, and neurons isolated from the brains of developing rats. J. Neurochem. 40, 120-127.

Wauquier A., Ashton D., Clincke G., and Fransen J. (1985) "Calcium entry blockers" as cerebral protecting agents: comparative activity in tests of hypoxia and hyperexcitability. Japan. I. Pharmacol. 38, 1-7.

Wong E. H. F., Kemp J. A., Priestley T., Knight A. R., Woodruff G. N., and Iversen L. L. (1986) The anticonvulsant MK-801 is a potent $N$-methyl- $D$ aspartate antagonist. Proc. Natl. Acad. Sci. USA 83, 7104-7108.

Yasuda H., Kishiro K., Izumi N., and Nakanishi M. (1985) Biphasic liberation of arachidonic and stearic acids during cerebral ischemia. J. Neurochem. 45, 168-172.

Yoshida S., Harik S. I., Busto R., Santiso M., Martinez E., and Ginsberg M. D. (1984) Free fatty acids and energy metabolites in ischemic cerebral cortex with noradrenergic depletion. J. Neurochem. 42, 711-717.

Yoshida S., Ikeda M., Busto R., Santiso M., Martinez E., and Ginsberg M. D. (1986) Cerebral phosphoinositide, triacylglycerol, and energy metabolism in reversible ischemia: origin and fate of free fatty acids. J. Neurochem. 47, $744-757$.

Yoshida S., Inoh S., Asano T., Sano K., Kubota M., Shimazaki H., and Ueta N. (1980) Effect of transient ischemia on free fatty acids and phospholipids in the gerbil brain. J. Neurosurg. 53, 323-331. 